\title{
Postural responses during the various frequencies of anteroposterior perturbation ${ }^{1}$
}

\author{
Kap Soo Han, Sun Hye Shin, Chang Ho Yu* and Tae Kyu Kwon* \\ Division of Biomedical Engineering, Chonbuk National University, 567 Baekje-daero, Deokjin-gu, \\ Jeonju-si, Jeonbuk 561-756, Republic of Korea
}

\begin{abstract}
This study investigated the characteristics of dynamic postural responses when subjects attempted to maintain an upright standing position on a support plate during continuous sinusoidal perturbation in the anterior-posterior direction. Fifteen healthy young subjects participated in the experiment. Body movement patterns during the perturbation were captured and analyzed using a 3D motion analysis system (APAS 3D motion analysis, Ariel Dynamics Inc.). Seven markers were attached on the subject's body to measure and analyze the motion patterns. The markers were positioned at the head, chest, hip, right knee, left knee, right ankle, and left ankle. Five different frequencies of motion were applied to the support surface: 0.1 , $0.5,1.0,1.5$, and $2.0 \mathrm{~Hz}$ with a $4-\mathrm{cm}$ path of motion at the base. The experiments measured dynamic postural responses in a condition were subjects had their eyes open. The results showed that the median frequency of the knee and ankle increased in all frequency bands. Following an increase in the frequency of the perturbation, the postural control strategy was changed from the ankle strategy to a combined strategy. These experimental results could be applied to the dynamic postural training for the elderly and to rehabilitation training for patients to improve their ability for postural control.
\end{abstract}

Keywords: Postural control, dynamic postural balance, motion base, horizontal translation

\section{Introduction}

Postural balance is the ability to maintain the center of gravity of the human body within the ground support area with minimal sway, and it is an important response that allows individuals to perform various daily activities $[1,2]$. Posture control to maintain balance requires the ability to correctly predict, detect, and encode perturbations [1]. A number of strategies are employed by the body in both static and dynamic conditions to keep the center of gravity (COG) within the base of support (BOS) to successfully maintain balance. Movements at the ankle joint (ankle strategy) are utilized in response to smaller, low-frequency perturbations; movements at the hip (hip strategy) are utilized in response to larger, high-frequency perturbations; and a stepping strategy is utilized to rapidly change the dimensions of the BOS in relation to the COG $[1,3]$.

\footnotetext{
${ }^{1}$ Authors contributed equally to this work.

*Corresponding authors: Chang Ho Yu, Division of Biomedical Engineering, Chonbuk National University, 567 Baekjedaero, Deokjin-gu, Jeonju-si, Jeonbuk 561-756, Republic of Korea. Tel.: +82-63-270-4323; Fax: +82-63-270-2247; E-mail: combo418@nate.com.

Tae Kyu Kwon, Division of Biomedical Engineering, Chonbuk National University, 567 Baekje-daero, Deokjin-gu, Jeonju-si, Jeonbuk 561-756, Republic of Korea. Tel.: +82-63-270-4066; Fax: +82-63-270-2247; E-mail: kwon10@jbnu.ac.kr.
}

0959-2989/14/\$27.50 @ 2014 - IOS Press and the authors. 
Balance control is necessary for postural safety and self-reliance, and the central nervous system (CNS) coordinates the position and movement of the body relative to external objects and environments based on neural information from the vestibular system, the somatosensory system, and the visual system [4]. The visual system senses external objects and environments; the vestibular system is involved in processing the spatial information of the head relative to gravity; and the somatosensory system senses the position and movements relative to the support surface and transfers these to the CNS $[5,6]$. Also, the somatosensory system is involved in processing the input information of the entire body from muscle receptors, joint receptors, and skin receptors on the skin and joints [7]. Muscle receptors provide compensatory information of muscle conditions and joint receptors and muscle receptors are involved in controlling the movement of the body together.

Recently, strategies that the body employs for to restore balance when falling have been studied, such as the ankle, hip, a combined strategy. The ankle strategy allows motion around the ankle joint without hip extension, and the hip strategy moves the hip forward and backward. Generally, these two strategies are combined to keep the balance of the body [8]. Nashner et al. reported that the ankle strategy mainly works by balancing the body while in a standing posture, and the combined strategy becomes involved in a case where rapid perturbations of the support area occur [5]. Winter described how the centers of mass of the head and body move in the same direction to compensate for perturbations in the ankle strategy, and the hip strategy is only involved in cases where balancing the body could not be completely restored through the ankle strategy [3].

Research regarding postural balance has been mainly conducted by limiting the input to certain sensing systems or by applying external perturbations using a force plate and then measuring the displacement, center of pressure (COP), and muscle activities necessary for the subject to keep the posture. Horak et al. reported that measurement of perturbations of the body on a fixed support area might not be sufficient for quantitative and qualitative evaluation of postural balance [8]. Therefore, Allum et al. and Commissaris et al. evaluated postural balance while perturbing the support area in the anteroposterior (AP) and mediolateral (ML) directions $[9,10]$. Müller et al. compared COP and muscle activation patterns to the perturbation of the support area in the AP direction [11]. Recently, research has been performed to understand postural balance and strategies by perturbing the support area, but the relationship between postural balance and strategies to dynamic perturbations of the support surface has not been investigated [12-14]. Therefore, the goal of this study is to investigate the characteristics of dynamic postural responses such as postural balance and strategies to balance the body with respect to the frequency of a perturbation of the support area. To this end, joint movements and body reactive characteristics were measured with respect to dynamic perturbations.

\section{Experimental methods}

\subsection{Motion base}

The experimental device was developed to investigate the postural balance as shown in Figure 1. The motion base (DSMP606, Simulink Co.) which has six actuators was used and it provides the function of excitation in the three directions of the translational motions by controlling the frequency and displacement at $30 \mathrm{~Hz}$ of sampling rate. The dimension of support surface was $1.55 \mathrm{~m}$ by $1.35 \mathrm{~m}$ and the subject was supposed to position on the center of the support surface. 

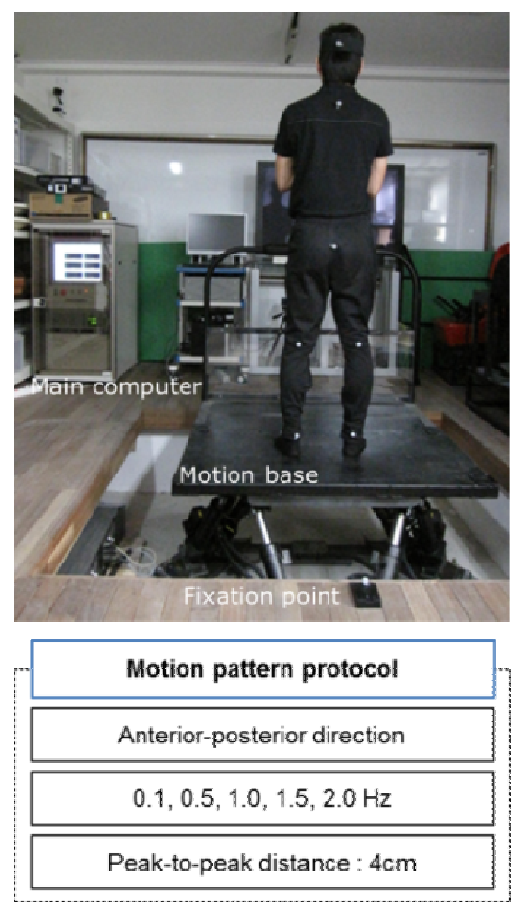

Fig. 1. Schematic of the dynamic postural control experiment using a motion base system.

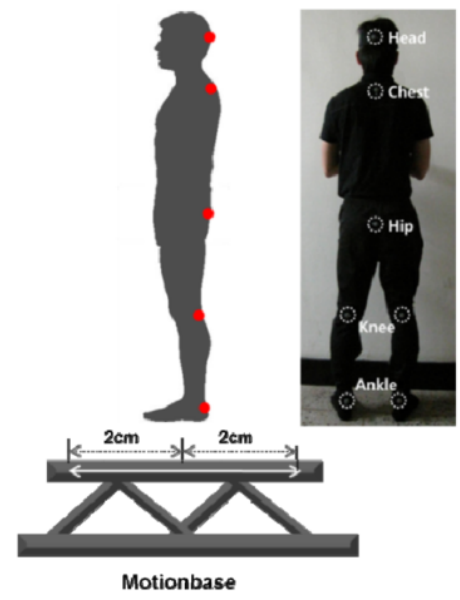

Fig. 2. Schematic of a subject on a support surface plate and marker positions.

\subsection{Motion capture analysis}

Two video cameras and seven markers (APAS 3D motion analysis, Ariel Dynamics Inc.) were used for 3-D motion capture of the motion of the body (Figure 2). A calibration tool and a fixation point were used to synchronize the time between real and video-taped motions. The video camera had a resolution of $720 \times 480$ and a $60 \mathrm{~Hz}$ frame refresh rate, and the motion data was recorded in the video recorder. The data for the analysis was obtained through a trimming process. The trimmed data from 
two cameras was converted into 3-D files using a digitizing transformation process and were then filtered for display in a graph format for the final analysis.

\subsection{Subjects}

In this study, fifteen healthy subjects (age, $25.8 \pm 1.3$ years; height, $174.4 \pm 3.4 \mathrm{~cm}$; weight, $67.8 \pm 5.7$ $\mathrm{kg}$ ) participated, and written informed consensus was collected in conformance with the Declaration of Helsinki (1963). None of the subjects had any history of musculoskeletal disease or neuromuscular control disorders, and they also had no prior experience with similar experiments.

\subsection{Experimental procedures and conditions}

Motion capture analysis was performed to measure the motion of the subject's body. The frequency of movement and the patterns of motion of the base varied in the AP direction of the support surface. Seven markers were attached, one on the head, chest, hip and one on each of both knees and both ankles. The representative applied excitation frequencies of $0.1,1.0$, and $2.0 \mathrm{~Hz}$, and the displacement patterns of the markers are shown in Figure 3. Information describing the movement of the support surface from low to high frequencies was shown to the subjects for their readiness, and a rehearsal was performed for the sake of their readiness and their safety. To remove the adaptation effect of the rehearsal, a random frequency of the excitation frequencies was applied in the actual experiment, and two minutes of relaxation were given to recover from muscular fatigue.

\subsection{Data analysis}

The experiment was conducted for 25 seconds, and five seconds from the beginning and the end of the data were trimmed out for the actual analysis. A low path filter, three times that of each oscillation

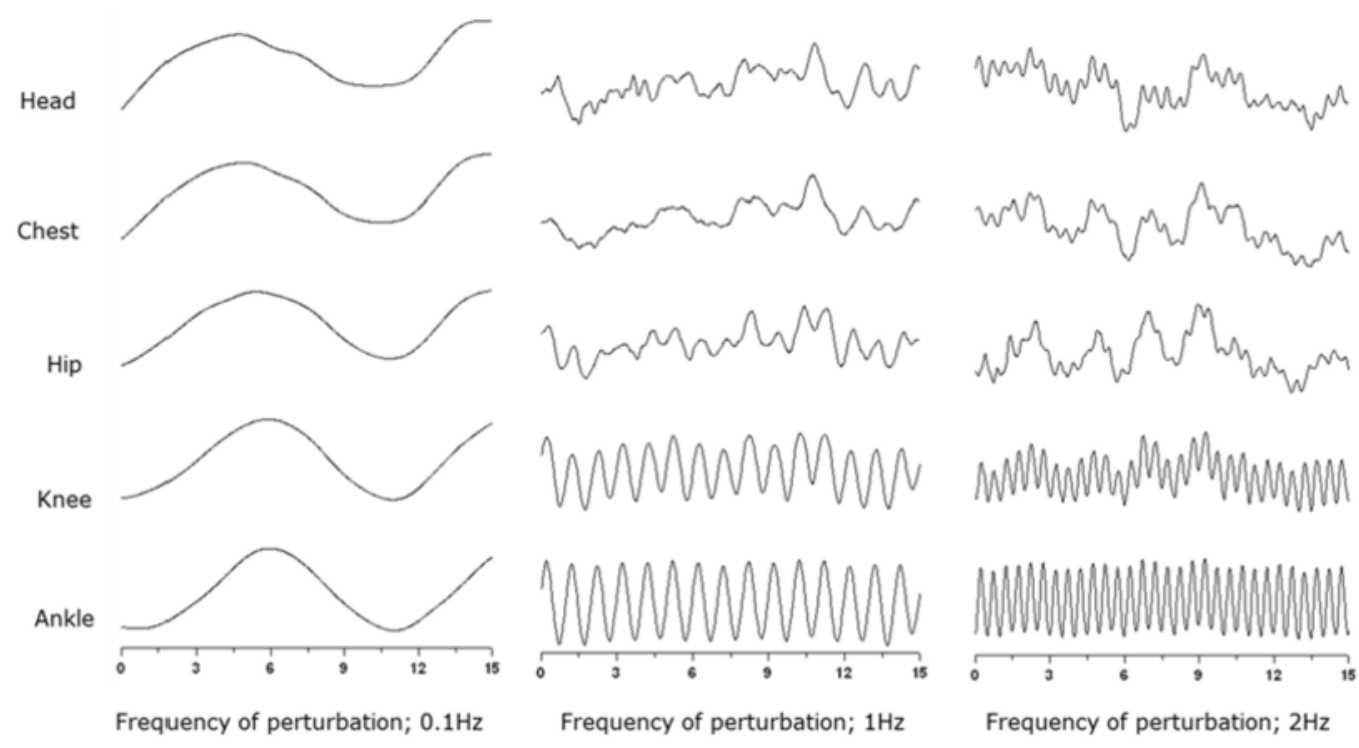

Fig. 3. Marker displacements according to different perturbation frequency bands. 
frequency, was used. The 3-D motion analysis from the markers on the head, chest, hip, both knees, and both ankles were analyzed, and the data of the two ankle parts were averaged. The median frequency from the entire perturbation frequency range, the absolute path, the relative path, and the correlation between the head and hip, chest and hip, knee and hip, ankle and hip, head and ankle, chest and ankle, and knee and ankle pairs were obtained by analyzing the movement of markers, as shown in Figure 1 . The median frequency $\left(f_{\text {median }}\right)$ was formulated as mathematical expression $(1)$, and $(f)$ indicated the power density spectrum of the EMG signal.

$$
\int_{0}^{f_{\text {median }}} s(f) d f=\int_{f_{\text {median }}}^{\infty} s(f) d f=\frac{1}{2} \int_{0}^{\infty} s(f) d f
$$

The median frequency was analyzed using a Fast Fourier Transform (FFT), and the relative path was obtained by calculating the distance between the path of the perturbation frequency on the support surface and the path of the corresponding markers. The coefficient of the correlation was obtained by calculating the correlation of each marker, where the coefficient ( $r$ ) always satisfies $-1 \leq r \leq 1$. Positive, negative, and no correlations were expressed as $r>0, r<0$ and $r=0$, respectively. Also, a coefficient close to 1 indicates a strong connection between two markers, and vice versa.

\section{Results and discussion}

\subsubsection{Marker movements}

Figure 4 shows the movement of the markers with respect to time, and the ankle path showed an almost identical trend in all excitation frequencies. The knee and ankle paths were similar to the excitation frequencies $(1.5 \mathrm{~Hz})$, but those of the head, chest, and hips were quite smaller than the path of the oscillation. As the frequency increased, the tendency for the head and the chest paths were harder to define. The median frequency was represented as a response of the dynamic postural balance (Figure 4) when the support surface was excited in the AP direction. As the frequency increased, the median frequency at the ankle and the knee also increased. This result indicates that the movement of the ankle and the knee correspond well to that of the support surface. The median frequency of the head, chest, and hips was not identical to that of the oscillation frequency in the cases where the frequency was higher than $0.5 \mathrm{~Hz}$.

\subsubsection{Postural strategy}

The response of the dynamic postural balance is shown in Figure 5 as the absolute distance value of each marker when the support surface was excited in the AP direction. In the cases with $0.1 \mathrm{~Hz}$ and $0.5 \mathrm{~Hz}$ perturbations, the absolute paths were similar for all frequencies except for the path of the ankle. This showed that the ankle strategy was primarily involved in compensating for the excitation of the plate and that it kept the body in balance. As the frequency of the perturbation increased, the absolute distance of the path of each part started to increase, and the difference of the paths with respect to each other became considerable. From a frequency of $1.0 \mathrm{~Hz}$, the absolute paths of the knee and the ankle were noticeably different from those of the other parts, and the head strategy also became involved in keeping balance. Generally, one or two strategies were enough to restore and sustain balance 
at a lower frequency of perturbation, but more strategies should be combined to restore postural balance when there is a perturbation with higher frequency.

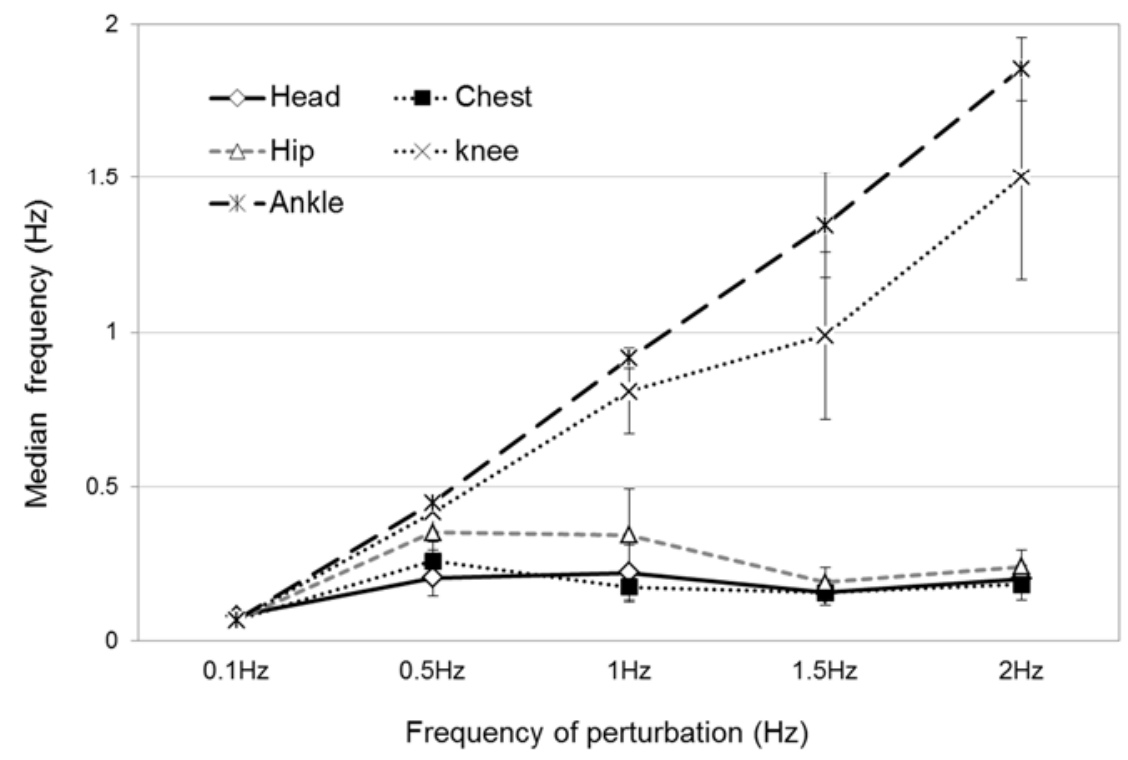

Fig. 4. Frequency response of each strategy with respect to different frequency bands.

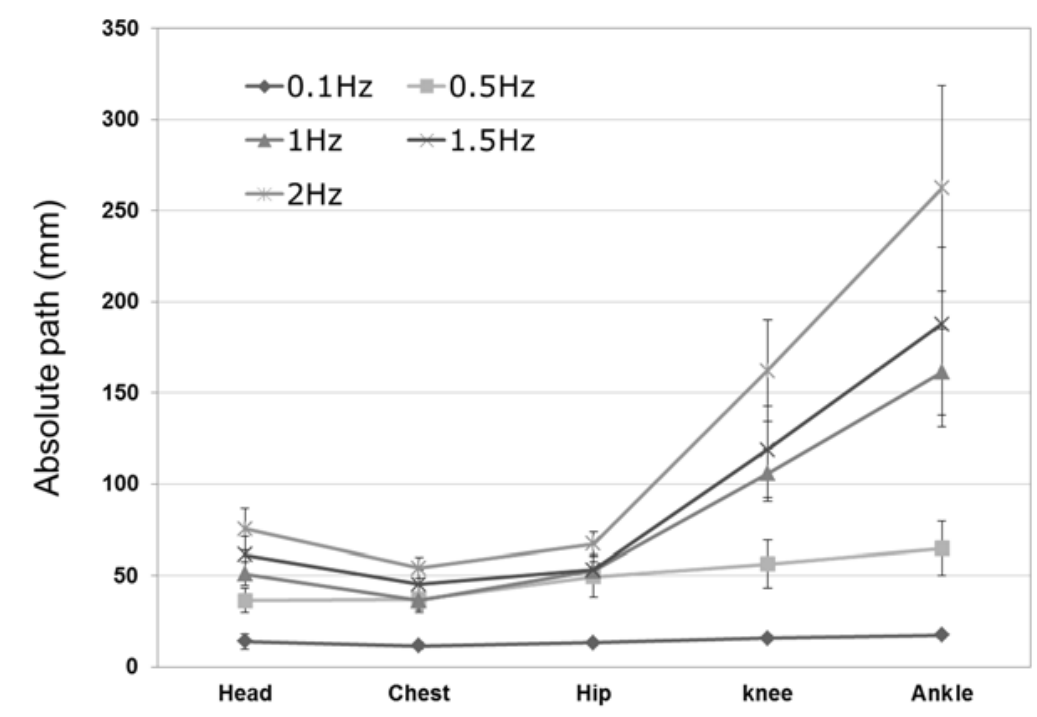

Fig. 5. Absolute path of each strategy with respect to the frequency of the perturbation. 


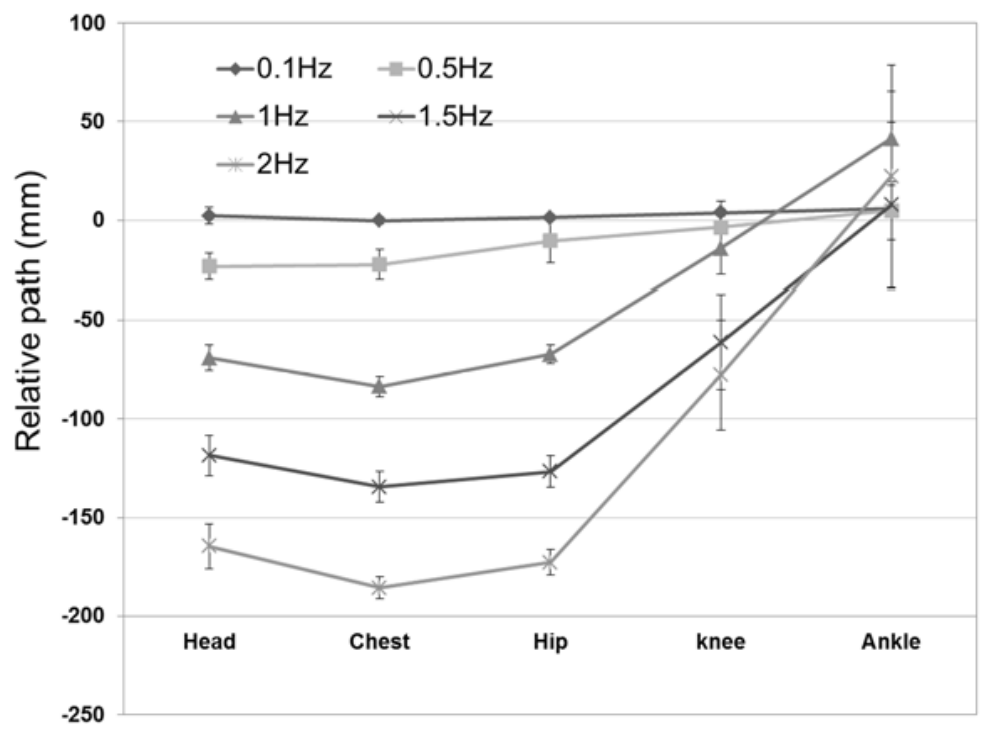

Fig. 6. Relative path of each strategy with respect to the frequency of the perturbation.

The relative path of the makers with respect to the support plate is given in Figure 6. When the distance between the marker and the support plate increased, the distance of the relative path also increased. This indicates that the body part was hardly involved in restoring postural balance from the perturbation. As shown in Figure 6, small relative paths were observed at lower perturbation frequencies, such as at $0.1 \mathrm{~Hz}$ and $0.5 \mathrm{~Hz}$, for all body parts. Hence, the head, chest, hip, knees, and ankle strategies were involved in restoring postural balance. However, as the perturbation frequencies increased $(1.0,1.5$, and $2.0 \mathrm{~Hz})$, the knees and ankles took major roles in maintaining postural balance, and the rest of the parts were only slightly involved.

\subsubsection{Correlation}

The data (Table 1) showed a correlation between the strategies with respect to the frequency of the perturbation. The head and hips showed a smaller correlation as the frequency increased over $1.0 \mathrm{~Hz}$, and the chest and hips were strongly correlated regardless of the perturbation frequencies. Hip-knee and hip-ankle pairs also showed decreasing correlation coefficients, but the hip-ankle pair showed a sharper decrease, indicating a weak correlation to postural balance. This implies that as the frequency increases, the strategy to maintain balance changes from an ankle related strategy to that of a total body component strategy. In the case of the head and ankles, a weak correlation was observed in all frequency ranges, except for $1.5 \mathrm{~Hz}$ and $2 \mathrm{~Hz}$. A similar trend was observed for the chest and ankle correlation. Knees and ankles showed a strong correlation throughout all perturbation frequencies. This implies that the knees and ankles are anatomically adjacent and work together to restore postural balance when compensating for an external perturbation $[3,8]$.

In summary, according to the frequency applied to the support plate, the head, chest, and hips were mainly affected by the median range of frequencies. To maintain postural balance, body parts moved along with the support plate at a lower range of frequencies (i.e., $0.1 \mathrm{~Hz}$ ), and the ankle strategy started to become involved at $0.5 \mathrm{~Hz}$. After $0.5 \mathrm{~Hz}$, the hips and ankles were involved in restoring postural balance while the upper parts, such as head and chest, were mainly still. This trend was more obvious as the perturbation frequency increased. Correlation between the parts became weaker as the 
Table 1

Changes in correlation on the frequency of perturbation. Values in parentheses are standard deviation

\begin{tabular}{llllll}
\hline & $0.1 \mathrm{~Hz}$ & $0.5 \mathrm{~Hz}$ & $1 \mathrm{~Hz}$ & $1.5 \mathrm{~Hz}$ & $2 \mathrm{~Hz}$ \\
\hline head/chest & $0.92(0.10)$ & $0.96(0.02)$ & $0.93(0.04)$ & $0.92(0.04)$ & $0.89(0.06)$ \\
head/hip & $0.77(0.15)$ & $0.75(0.08)$ & $0.56(0.13)$ & $0.45(0.18)$ & $0.46(0.10)$ \\
head/knee & $0.64(0.17)$ & $0.53(0.13)$ & $0.26(0.13)$ & $0.33(0.20)$ & $0.30(0.08)$ \\
head/ankle & $0.56(0.18)$ & $0.28(0.15)$ & $0.01(0.07)$ & $0.24(0.15)$ & $0.18(0.05)$ \\
chest/hip & $0.89(0.06)$ & $0.87(0.04)$ & $0.72(0.08)$ & $0.66(0.13)$ & $0.65(0.08)$ \\
chest/knee & $0.77(0.09)$ & $0.65(0.12)$ & $0.36(0.11)$ & $0.39(0.18)$ & $0.32(0.09)$ \\
chest/ankle & $0.68(0.12)$ & $0.41(0.15)$ & $0.06(0.07)$ & $0.18(0.16)$ & $0.10(0.05)$ \\
hip/knee & $0.96(0.02)$ & $0.88(0.07)$ & $0.76(0.05)$ & $0.71(0.08)$ & $0.57(0.09)$ \\
hip/ankle & $0.9(0.05)$ & $0.68(0.13)$ & $0.42(0.08)$ & $0.3(0.14)$ & $0.12(0.10)$ \\
knee/ankle & $0.98(0.01)$ & $0.89(0.06)$ & $0.86(0.05)$ & $0.8(0.06)$ & $0.79(0.05)$ \\
\hline
\end{tabular}

frequency of the perturbation increased, except for the knee and ankle joints. The correlation of the head-ankle and chest-ankle pairs sharply decreased in the cases where the frequency was higher than $0.5 \mathrm{~Hz}$. At a lower range of perturbation frequencies, the ankle strategy was mainly activated, but the head and chest were stabilized, and the hips and knees were involved in maintaining postural balance as the perturbation frequency increased.

\section{Conclusion}

In this study, the ability of the joints of the body to maintain dynamic postural balance was investigated as a perturbation was applied to the support plate. The response of normal subjects in terms of an external perturbation was shown in this study. Therefore, these results can be used as a standard to analyze the response according to balance and posture of elderly individuals and those with a disability. Also the experimental mechanism can be used as part of training and rehabilitation programs to restore the ability of dynamic postural balance.

\section{Acknowledgement}

This research was financially supported by the Ministry of Trade, Industry and Energy (MOTIE) and Korea Institute for Advancement of Technology (KIAT) through the Promoting Regional Specialized Industry (R0002430) and partially supported by SME Tech Convergence Development Project (No. S2023416). 


\section{References}

[1] F.B. Horak, C.L Shupert and A. Mirka, Components of postural dyscontrol in the elderly: A review, Neurobiology of Aging 10 (1989), 727-738.

[2] A. Shumway-Cook, Motor Control: Theory and Practical Applications, 2nd ed., Williams \& Wilkins, Baltimore, 2001, pp. 4-62.

[3] H. Cohen, C.A. Blatchly and L.L. Gombash, A study of the clinical test of sensory interaction and balance, Physical Therapy 73 (1993), 346-354.

[4] L.M. Nashner and G. McCollum, The organization of human postural movements: A formal basis and experimental synthesis, Behavioral and Brain Science 8 (1985), 135-150.

[5] G.E. Riccio and T.A. Stoffregen, Affordances as constraints on the control of stance, Human Movement Science 7 (1988), 265-300.

[6] A. Shumway-cook, Motor Control: Theory and Practical Applications, 1st ed., Williams \& Wilkins, Baltimore, 1995, pp. 119-206.

[7] F.B. Horak, Clinical assessment of balance disorder, Gait \& Posture 6 (1997), 76-48.

[8] D.A. Winter, Anatomy Biomechanics and Control of Balance during Standing and Walking, $1^{\text {st }}$ ed., Waterloo Biomechanics Inc., Waterloo, 1995, pp. 5-32.

[9] J.H. Allum, Organization of stabilizing reflex responses in tibialis anterior muscles following ankle flexion perturbations of standing man, Brain Research 264 (1983), 297-301.

[10] D.A. Commissaris, P.H. Nieuwenhuihzen, S. Overeem, A. de Vos, J.E. Duysens and B.R. Bloem, Dynamic posturography using a new movable multidirectional platform driven by gravity, Journal of Neuroscience Methods 113 (2003), 73-84.

[11] M.L. Müller and M.S. Redfern, Correlation between EMG and COP onset latency in response to a horizontal platform translation, Journal of Biomedical 37 (2004), 1573-1581.

[12] K.S. Tae and Y.H. Kim, Balance recovery mechanism against anterior perturbation during standing, Journal of Biomedical Engineering Research 24 (2003), 435-442.

[13] Y.K. Shin, M.A. Fard, H. Inooka and I.H. Kim, Human postural dynamics in response to the horizontal vibration, International Journal of Control, Automation, and Systems 14 (2006), 325-332.

[14] S.M. Henry, J. Fung and F.B. Horak, Control of stance during lateral and anterior/posterior surface translations, IEEE Transactions on Rehabilitation Engineering 6 (1998), 32-42. 